\title{
Supporting Action Planning for Sedentary Behavior Change by Visualizing Personal Mobility Patterns on Smartphone
}

\author{
Yunlong Wang \\ Human-Computer Interaction Group \\ University of Konstanz \\ Konstanz, Germany \\ yunlong.wang@uni-konstanz.de
}

\author{
Ahmed Fadhil \\ Centro Ricerche GPI \\ Fondazione Bruno Kessler (FBK) \\ Trento, Italy \\ fadhil@fbk.eu
}

\author{
Harald Reiterer \\ Human-Computer Interaction Group \\ University of Konstanz \\ Konstanz, Germany \\ harald.reiterer@uni-konstanz.de
}

\begin{abstract}
Scientific evidence has shown that long-term sedentary behaviour is detrimental to human health. Therefore, a trend appears in the field of healthy lifestyle promotion that more attention is drawn to sedentary behaviour rather than only physical activity. However, technology-based mobile health intervention tools targeting reducing sedentary behaviour are still lacking. This paper aims to explore a solution for sedentary behaviour change through supporting action planning. Action planning can not only bridge the intention-behavior gap in controlled motivation processes, but also enforce the cue-behavior association in unconscious processes. We present a smartphone-based personal mobility pattern visualization, with which we expect the users can make better action plans. The interactive visualization integrates temporal and spatial patterns of personal sedentary and walking behaviour, to provide explicit hints on when, where, and how to reduce sedentary behaviour and increase daily steps. We also present our experimental design to evaluate the visualizationbased intervention tool.
\end{abstract}

\section{CCS CONCEPTS}

- Human-centered computing $\rightarrow$ Visualization design and evaluation methods

\section{KEYWORDS}

Action planning, sedentary behavior, mobile health intervention, mobility pattern visualization

\section{INTRODUCTION}

Good intentions are more likely to be translated into action when people develop success scenarios and preparatory strategies of approaching a difficult task [24]. According to behavioral theories $[1,24,27]$, action planning plays an important role in health behavior change as it bridges the intention-behavior gap. For example, a fitness coach in a gym helps his clients to make fitness plans to achieve their goals. Unfortunately, many physical activity applications (apps) do not provide the function of action planning, even in top-ranked apps [6]. Fitness apps usually ask users to set a goal (e.g., 10,000 steps per day) and track users' performance, but without providing instructions on how to achieve the goal or supporting action planning. A recent review of behavior change interventions with fitness promoting technologies on sedentary adults also shows that promising strategies, including action planning, are not typically included in fitness technology devices or apps [26].

In this paper, we will present a smartphone-based personal mobility pattern visualization tool focusing on supporting action planning for sedentary behaviour change. We also propose an experimental study to evaluate the efficacy of the proposed tool. The novelty of our approach is that it shows the users their sedentary and non-sedentary behavior in both temporal and spatial patterns. In other words, it illustrates where, when, and how much are the users physically active or not. We now define all the places or areas a person stays for a period as Places of Interest (POIs). Then the POIs and the transitions among them contain all the information of our mobility, where our daily walking steps are embedded. We expect our visualization tool can inspire the users to better understand their sedentary behavior, make action plans for behavior change, and finally improve their sedentary behavior.

We design and conduct the study following a holistic approach [31] to mobile health interventions, including understanding the specific behavior using behavioral theories, targeting the user group and outcome, designing and implementing the intervention tool, evaluating, and refining. The remainder of the paper is organized as follows. Sect. 2 shows the background on sedentary behavior and action planning, which is the understanding step. 


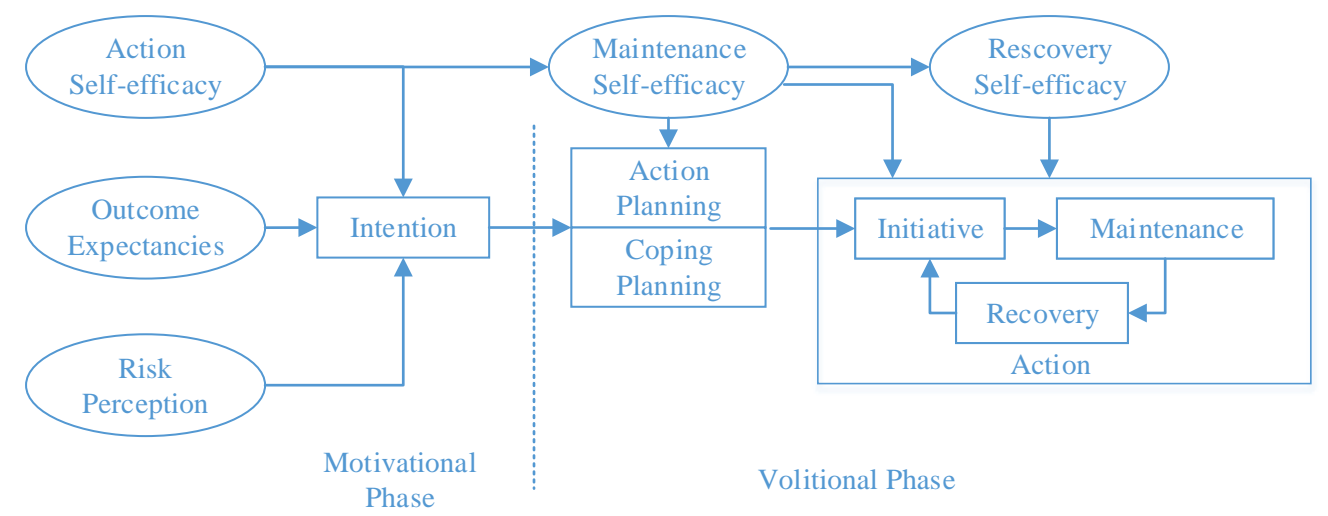

Figure 1: The model of Health Action Process Approach (HAPA) [24]. Action planning is a mediator between the intervention and the action according to HAPA.

Following, we discuss the related work in Sect.3. In Sect. 4, we report our targeted user group and outcome, the designed intervention tool, as well as our study plan. Finally, we conclude this paper and illustrate our future work.

\section{BACKGROUND}

\subsection{Sedentary Behavior}

Prolonged sedentary behavior (SB) has been found to be related to many chronic diseases, such as cardiovascular diseases, diabetes, obesity, and high blood pressure [3,20]. Industry development and urbanization promotes sedentary lifestyle in general. As mobile technologies, especially smartphone, is pervasive in our daily life, increasing research has focused on mobile health technology. To reduce sedentary behavior, one can simply change to standing position, walking, or using physical activity machines (e.g., treadmills or pedal machine ) [14, 22]. Machines require extra space and are always costly and noisy, so they are not suitable in many cases. In our study, we encourage users to walk more as a mean to reduce prolonged sedentary behavior, because: (1) walking, as a kind of light intensity physical activity, shows more benefit than standing [22]; (2) it needs no extra machines; (3) it can be measured using the pedometer on smartphones. Inspired by Fitbit $250+$ Steps, we regard an hour with more than 250 steps as an active hour. The goal is to reduce sedentary (inactive) hours and empower sedentary adults to increase energy expenditure during waking time.

\subsection{Action Planning}

Action planning for a target behavior includes specific situation parameters ("when", "where") and a sequence of actions ("how") [24]. Behavior change interventions incorporate action planning by asking people to create a detailed plan describing when, where, and how they would engage or reduce the target behavior.

Action planning can not only bridge the intention-behavior gap (as shown in Figure 1) in controlled motivation processes [24, 27], but also enforce the cue-behavior association in unconscious processes [16]. Drawing on the dual-process model [11], Conroy et al. found that SB was regulated by both automatic and controlled motivational processes [5]. Thus, we believe action planning should be an irreplaceable element in sedentary behavior change interventions.

As rooted in behavioral theories, the well-known taxonomy of health behavior change - Behavior Change Techniques (BCTs) also includes action planning [17]. It is defined as prompting detailed planning of performance of the behavior (must include at least one of context, frequency, duration, and intensity) in BCTs.

From the perspective of Persuasive Technology (PT) in HCI, a design principle - tunneling - defined as guiding users through a process or experience is also relevant to action planning $[12,19]$. However, only when the process explicitly includes at least one situation parameter ("when" and "where") and one action sequence ("how"), we regard the system as supporting action planning. We mentioned BCTs and PT because they are frequently used to code intervention elements in systematic reviews of mobile health interventions [2, 7, 9, 13, 15, 25, 34].

\section{RELATED WORK}

\subsection{SB Reduction Strategies}

Based on the review on PT in reducing prolonged sedentary behavior at work [34], the deployment of action planning is very limited in the selected randomized controlled trials. This finding is in line with another review on sedentary behavior interventions using BCTs [25]. Although most of the selected studies did not explicitly ask users to create their own action plan over time, they provided participants suggestion on how to interrupt (e.g., short standing breaks [4]) and reduce sedentary behavior (e.g., using standing desk [10], walking hallways, stairs, outdoor [28]), they. In the study conducted by Puig-Ribera et al. [21], several interesting strategies were provided targeting sedentary office employees in university, including: (1) moving rather than sitting during lectures and seminars; (2) not sitting to take phone calls; (3) choosing the "longest route" to go to another Department within the campus; (4) walking to work whenever possible; (5) 
taking walks after lunch. We use these strategies to compare them with the action plans made by the participants in our study.

\subsection{Mobility Pattern Visualization}

Mobility Pattern Visualization (MPV) aims to aid people to gain insights on human mobility. Many fitness mobile apps, such as Google Fit, can visualize users' physical activity with rich information including time, location, and activity. However, the visualization of the tracked mobility data is quite simple, e.g., hourly activity summary is not supported. Most sedentary behavior prevention apps only provide a dedicated timer, while visualization targeting sedentary behavior is seldom among top fitness apps. Fitbit ${ }^{1}$ introduced a $250+$ Steps visualization (as shown in Figure 2) in a recent version. Users can see in which hour(s) they achieve the 250 steps goal or not from this visualization. Drawing on this idea, our visualization aims to provide more context details on users' sedentary behavior.

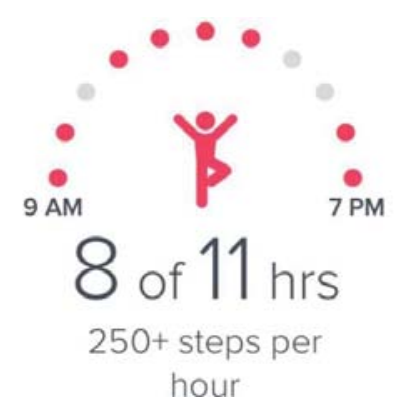

Figure 2: Fitbit 250+ Steps per hour visualization. The red dots mean the user took more than 250 steps in the corresponding hours.
We find no sedentary intervention mobile app that incorporates visualization and action planning. However, some studies targeting physical activity proposed innovative and effective visualizations (e.g., [23, 29]). MyBehavior is a smartphone app that can visualize mobility patterns using a map and automatically generate physical activity suggestions based on the activity levels in visited places of a user, e.g., small walk near Campus Road [23]. The results of a 14-week study showed statistically significant increases in physical activity when using MyBehavior compared to the control condition, where the users were provided a prepared suggestion list of physical activities. Another work by Tong et al. [29] compared the effectiveness of three physical activity visualizations (i.e., the bar chart, ring map, and the virtual pet calendar) on users' perception and task performance. The results showed no significant difference of task performance among the three visualization groups, while the perceived readability and comprehension were better perceived in bar chart than in other visualizations. Based on the lessons learned from $[23,29]$, we integrate a map with a bar chart in our visualization tool.

\section{VISUALIZATION TOOL}

\subsection{User Interface}

Instead of providing users simple suggestions on action planning, we intend to encourage users to independently decide their plans using the visualization. There are two considerations underlying: (1) to explore the power of our visualization on action planning, and (2) to leverage the human creativity to gain more user engagement $[18,32]$.

The user interface shown in Figure 3 is a mockup, aiming to illustrate the idea of mobility pattern-based visualization for
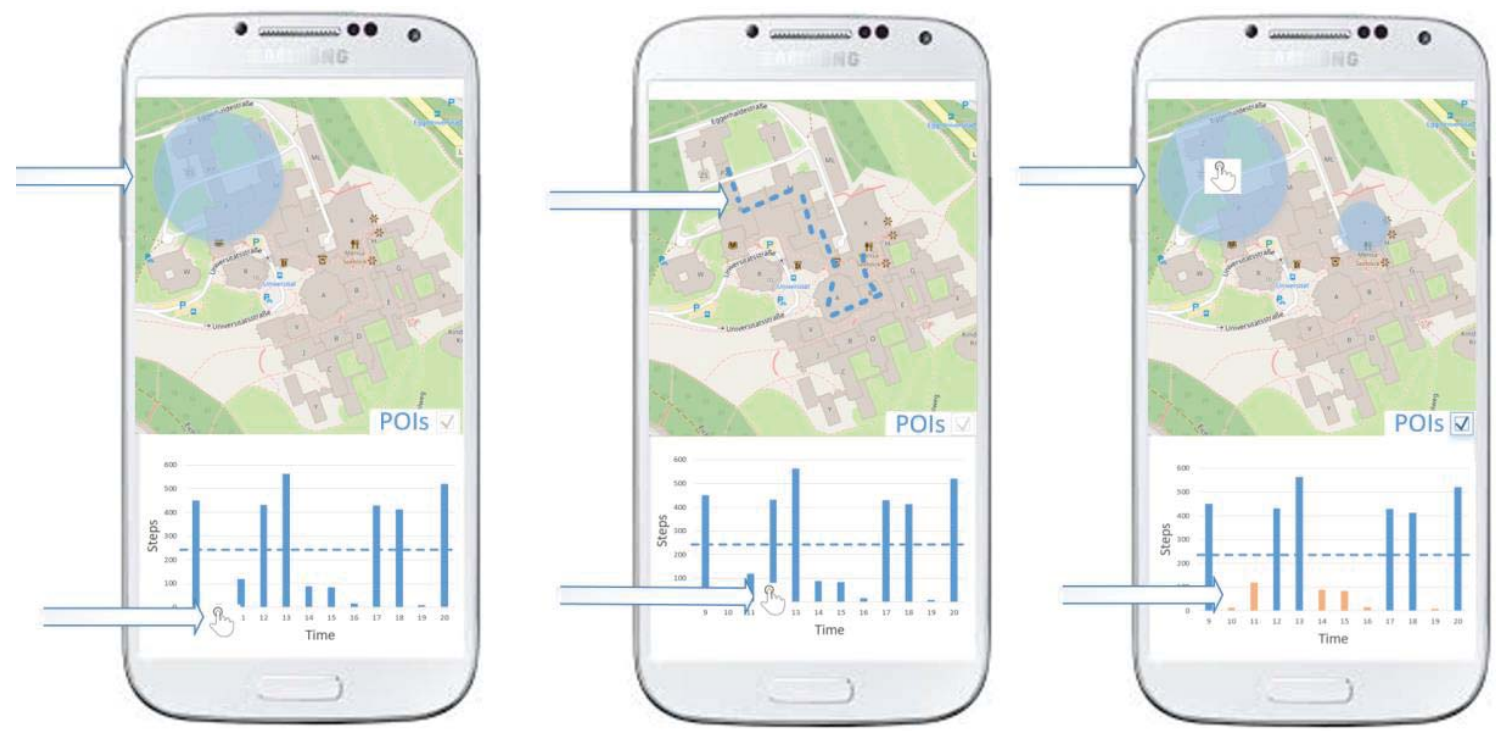

Figure 3: The interface of our mobility pattern visualization app. The corresponding POI shows up on the map when a sedentary hour bar is clicked in the bar chart (left). The corresponding route shows up on the map when an active hour bar is clicked in the bar chart (middle). The corresponding hours bars are highlighted when a POI is clicked on the map (right). 
sedentary and active hours. The interactive interface contains a map and a bar chart. The POIs (the blue circle on the map in Figure 3) are extracted using the clustering method proposed in [33]. The size of the POI circle reflects the relative stay duration of the user. Using the bar chart, a user can see (1) the active hour(s) with more than 250 steps and the corresponding routes on the map once the bar is clicked (the positive feedback), and (2) the sedentary hour(s) and the corresponding POI(s) once the bar is clicked (negative feedback). While the bar chart illustrates temporal patterns, the map demonstrates spatial patterns. On the map, all POIs show up when the POIs option is selected. By clicking on one POI, the corresponding bars will be highlighted, showing if this POI is "dangerous" or not. As shown in Figure 3 (left), the user is always sedentary (less than 250 steps per hour) in the selected POI. Therefore, it is intuitive that the user would plan to walk more during the highlighted hours in this POI. The user may choose the route shown in Figure 3 (middle) to achieve the 250 steps goal because it is feasible in the user' walking history. Via all patterns based on temporal ("when"), spatial ("where"), and walking routes ("how") information, we expect to support users to easily learn their mobility patterns and make action plans.

\subsection{Implementation}

The app is implemented with Android framework. Underlying the user interface, it also contains a background service that oversees data collection. The data collection service is built on the work [30], where we use a state-based method to efficiently collect the mobility data. A state is a time-stamped information collection containing all the related context, e.g., location, Wi-Fi connection, physical activity, and steps. In this app, we detect users' physical activity every five seconds to ensure a high temporal resolution. To avoid redundancy, only when the current physical activity is NOT "still", we query and record location and steps. Because all the information in stationary ("still") state keeps unchanged and users are stationary in most of their time, especially for sedentary people. Thus, the location query, that drains the battery power, can be reduced to save much battery power. For privacy consideration, we only record the collected data on users' smartphone.

The APIs and libraries used in this app include Google Activity Recognition $\mathrm{API}^{1}$, Google Fused Location Provider $\mathrm{API}^{2}$, Google Fitness $\mathrm{API}^{3}$, Google Maps $\mathrm{API}^{4}$, MPAndroidChart ${ }^{5}$, and Realm ${ }^{6}$.

\section{EXPERIMENTAL DESIGN}

\subsection{Hypotheses}

\footnotetext{
${ }^{1}$ https://developers.google.com/location-context/activity-recognition/ ${ }^{2}$ https://developers.google.com/location-context/fused-location-provider/

${ }^{3}$ https://developers.google.com/fit/android/history

${ }^{4}$ https://developers.google.com/maps/

${ }^{5}$ https://github.com/PhilJay/MPAndroidChart

${ }^{6}$ https://realm.io/
}

(H1) The proposed mobility pattern visualization app can support action planning for reducing SB.

(H2) The proposed mobility pattern visualization app can enable behavior change on reducing SB.

\subsection{Participants and Procedure}

The participants will be recruited through the social network of the authors. All the participants should have the intention to reduce sedentary behavior and increase daily steps, while without any plan or action to do so. The users' stage of behavior change will be evaluated using Health Action Process Approach (HAPA) questionnaire [24].

\section{Session 1}

(1) An experimenter briefly introduces the detrimental effect of long-term sedentary behavior to participants.

(2) An experimenter helps participants to install the app on their smartphones and start the app to collect data.

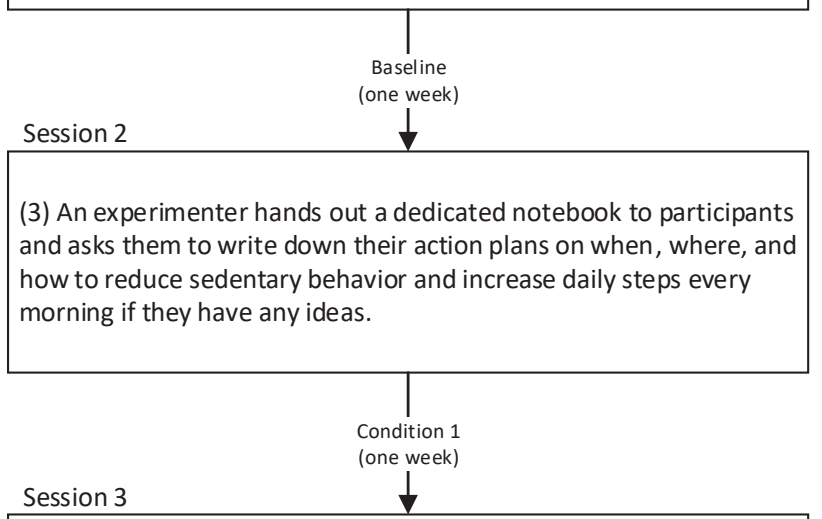

(4) An experimenter guides participants to open the mobility pattern visualization in the app and demonstrates how to interact with it . The participants are asked to use the app as they like.

(5) An experimenter asks participants to write down their action plans on when, where, and how to reduce sedentary behavior and increase daily steps every morning if they have any ideas.

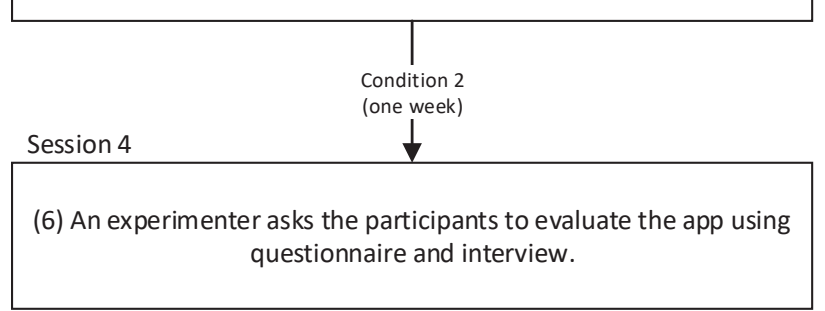

Figure 4: The study procedure following single-case experimental design.

We adopt a single-case experimental design with one baseline and two treatment conditions [8]. Each participant in a single-case study serves her or his own control, and the assessments are 
required to be measured repeatedly to achieve sufficient statistical power. These properties are suitable for preliminary evaluation of technology-based health interventions. The details of our study procedure are shown in Figure 4. During baseline, the app is only used to collect mobility data, without showing the visualization (as shown in Figure 3) to the participants. Because asking participants to make action plans is an intervention by itself, we use two treatment conditions (with or without our visualization) to access the effect of the visualization on both action planning (H1) and behavior change (H2). In other words, the intervention in session 2 is asking participants to make action planning, while the intervention in session 3 is asking participants to use our app and make action planning. We expect participants to have better action planning in session 3 than in session 2. Meanwhile, the participants' sedentary behavior is expected to be reduced gradually in section 2 (condition 1) and section 3 (condition 2), compared to session 1 (baseline).

\subsection{Data Collection}

(1) Participants' demographic data (i.e., age, gender, education, health status) will be collected by questionnaire in session 1 .

(2) Participants' mobility data, including spatial-temporal data and steps, and their interaction with the visualization will be collected automatically by the app.

(3) The action plans will be manually recorded by the participants and collected by the experimenter in session 4 .

(4) User experience will be evaluated using User Experience Questionnaire (UEQ) in session 4.

\subsection{Data analysis}

To test $\mathrm{H} 1$, both quantitative and qualitative evaluation will be carried out to compare the action plans made by the participants in the two conditions, which is based on the elements of action planning ("when", "where", and "how"). The data of sedentary behavior (inactive hours) and daily steps will be used to test $\mathrm{H} 2$. The analysis, based on the results of UEQ and interview the last session, will serve to generate discussions on open questions.

\section{DISCUSSION}

The scope of the study is exploring the power of a smartphone based-visualization on supporting users' action planning for sedentary behavior change. The targeted population would be individuals who intend to change their sedentary lifestyle. Our study can potentially provide implications on user interface design of smart coaching systems. The present visualization may serve as a sedentary behavior change technique to help people prevent the escalation of their condition into corresponding chronic diseases.

\section{CONCLUSIONS}

In this paper, we presented a smartphone-based personal mobility pattern visualization tool to support action planning for reducing sedentary behavior and increasing daily steps. The background on sedentary behavior related behavioral theories are introduced, while the related work on sedentary behavior reduction interventions and human mobility pattern visualization are reviewed. Finally, we proposed an experimental design to evaluate the proposed visualization tool. The described study is under preparation as of writing this paper. We will report the study evaluation and results in future publications.

\section{ACKNOWLEDGMENTS}

This work was partially supported by SMARTACT. Y. Wang acknowledges the financial support from the China Scholarship Council (CSC).

\section{REFERENCES}

[1] Ajzen, I. 1985. From intentions to actions: A theory of planned behavior. Action control: From cognition to behavior. (1985), 11-39. DOI:https://doi.org/10.1007/978-3-642-69746-3_2.

[2] Belmon, L.S., Middelweerd, A., te Velde, S.J. and Brug, J. 2015. Dutch Young Adults Ratings of Behavior Change Techniques Applied in Mobile Phone Apps to Promote Physical Activity: A Cross-Sectional Survey. JMIR mHealth and uHealth. 3, 4 (Nov. 2015), e103. DOI:https://doi.org/10.2196/mhealth.4383.

[3] Chastin, S.F.M., Palarea-Albaladejo, J., Dontje, M.L. and Skelton, D.A. 2015. Combined effects of time spent in physical activity, sedentary behaviors and sleep on obesity and cardio-metabolic health markers: A novel compositional data analysis approach. PLoS ONE. 10, 10 (2015), 1-21. DOI:https://doi.org/10.1371/journal.pone.0139984.

[4] De Cocker, K., De Bourdeaudhuij, I., Cardon, G. and Vandelanotte, C. 2015. Theory-driven, web-based, computer-tailored advice to reduce and interrupt sitting at work: development, feasibility and acceptability testing among employees. BMC public health. 15, 1 (Sep. 2015), 959. DOI:https://doi.org/10.1186/s12889-015-2288-y.

[5] Conroy, D.E., Maher, J.P., Elavsky, S., Hyde, A.L. and Doerksen, S.E. 2013. Sedentary behavior as a daily process regulated by habits and intentions. Health psychology: official journal of the Division of Health Psychology, American Psychological Association. 32, 11 (Nov. 2013), 1149-57. DOI:https://doi.org/10.1037/a0031629.

[6] Conroy, D.E., Yang, C.-H. and Maher, J.P. 2014. Behavior Change Techniques in Top-Ranked Mobile Apps for Physical Activity. American Journal of Preventive Medicine. 46, 6 (2014), 649-652. DOI:https://doi.org/10.1016/j.amepre.2014.01.010.

[7] Crane, D., Garnett, C., Brown, J., West, R. and Michie, S. 2015. Behavior Change Techniques in Popular Alcohol Reduction Apps. Journal of Medical Internet Research. 17, 5 (May 2015), e118. DOI:https://doi.org/10.2196/jmir.4060.

[8] Dallery, J., Cassidy, R.N. and Raiff, B.R. 2013. Single-case experimental designs to evaluate novel technology-based health interventions. Journal of medical Internet research. 15, 2 (Feb. 2013), e22. DOI:https://doi.org/10.2196/jmir.2227.

[9] Dombrowski, S.U., Sniehotta, F.F., Avenell, A., Johnston, M., MacLennan, G. and Araújo-Soares, V. 2012. Identifying active ingredients in complex behavioural interventions for obese adults with obesity-related co-morbidities or additional risk factors for co-morbidities: a systematic review. Health

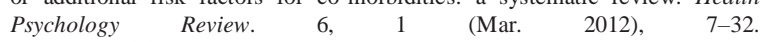
DOI:https://doi.org/10.1080/17437199.2010.513298.

[10] Donath, L., Faude, O., Schefer, Y. and Roth, R. 2015. Repetitive daily point of choice prompts and occupational sit-stand transfers, concentration and neuromuscular performance in office workers: an RCT. International journal of environmental research and public health. 2015 Apr 20;12(4):4340-53. (2015).

[11] Evans, J.S.B.T. (Ed) and Frankish, K. (Ed) 2009. In two minds: Dual processes and beyond. Oxford University Press.

[12] Fogg, B.J. 2003. Persuasive technology: using computers to change what we think and do. Morgan Kaufmann Publishers.

[13] Hamari, J., Koivisto, J. and Pakkanen, T. 2014. Do persuasive technologies persuade? - A review of empirical studies. Lecture Notes in Computer Science (including subseries Lecture Notes in Artificial Intelligence and Lecture Notes in Bioinformatics). 8462 LNCS, (2014), 118-136. DOI:https://doi.org/10.1007/978-3-319-07127-5_11.

[14] Henson, J., Davies, M.J., Bodicoat, D.H., Edwardson, C.L., Gill, J.M.R., Stensel, D.J., Tolfrey, K., Dunstan, D.W., Khunti, K. and Yates, T. 2015. Breaking Up Prolonged Sitting With Standing or Walking Attenuates the Postprandial Metabolic Response in Postmenopausal Women: A Randomized Acute Study. Diabetes Care. 39, 1 (2015).

[15] Kaptein, M., Markopoulos, P., De Ruyter, B. and Aarts, E. 2015. Personalizing 
persuasive technologies: Explicit and implicit personalization using persuasion profiles. International Journal of Human Computer Studies. 77, (2015), 3851. DOI:https://doi.org/10.1016/j.ijhcs.2015.01.004.

[16] Maher, J.P. and Conroy, D.E. 2015. Habit Strength Moderates the Effects of Daily Action Planning Prompts on Physical Activity but Not Sedentary Behavior. Journal of Sport and Exercise Psychology. 37, 1 (2015), 97-107.

[17] Michie, S., Richardson, M., Johnston, M., Abraham, C., Francis, J., Hardeman, W. Eccles, M.P., Cane, J. and Wood, C.E. 2013. The behavior change technique taxonomy (v1) of 93 hierarchically clustered techniques: Building an international consensus for the reporting of behavior change interventions. Annals of Behavioral Medicine. 46, 1 (2013), 81-95. DOI:https://doi.org/10.1007/s12160-013-9486-6.

18] Norton, M.I., Mochon, D. and Ariely, D, 2012. The IKEA effect: When labor leads to love. Journal of Consumer Psychology. 22, 3 (2012), 453-460. DOI:https://doi.org/10.1016/j.jcps.2011.08.002.

[19] Oinas-kukkonen, H. and Harjumaa, M. 2009. Persuasive Systems Design : Key Issues, Process Model, and System Features Persuasive Systems Design: Key Issues, Process Model , and System Features. Communications of the Association for Information Systems. 24, 1 (2009).

[20] Owen, N., Sugiyama, T., Eakin, E.E., Gardiner, P.A., Tremblay, M.S. and Sallis, J.F. 2011. Adults' Sedentary Behavior. American Journal of Preventive $\begin{array}{llll}\text { Medicine. } & 41, \quad 2011), & \text { (Aug. }\end{array}$ DOI:https://doi.org/10.1016/j.amepre.2011.05.013.

[21] Puig-Ribera, A., Bort-Roig, J. and González-Suárez, A. 2015. Patterns of impact resulting from a "sit less, move more"web-based program in sedentary office employees. PloS one. (2015).

[22] Pulsford, R.M., Blackwell, J., Hillsdon, M. and Kos, K. 2016. Intermittent walking, but not standing, improves postprandial insulin and glucose relative to sustained sitting: A randomised cross-over study in inactive middle-aged men. Journal of science and medicine in sport. (Aug. 2016). DOI:https://doi.org/10.1016/j.jsams.2016.08.012

[23] Rabbi, M., Aung, M.H., Zhang, M. and Choudhury, T. 2015. MyBehavior: automatic personalized health feedback from user behaviors and preferences using smartphones. Ubicomp '15, September 7-11, 2015, Osaka, Japan. (2015), 707-718. DOI:https://doi.org/10.1145/2750858.2805840.

[24] Schwarzer, R. 2008. Modeling health behavior change: How to predict and modify the adoption and maintenance of health behaviors. Applied Psychology. 57, 1 (2008), 1-29. DOI:https://doi.org/10.1111/j.14640597.2007.00325.x.

[25] Stephenson, A., McDonough, S.M., Murphy, M.H., Nugent, C.D, and Mair, J.L. 2017. Using computer, mobile and wearable technology enhanced interventions to reduce sedentary behaviour: a systematic review and metaanalysis. International Journal of Behavioral Nutrition and Physical Activity. 14, 1 (Dec. 2017), 105. DOI:https://doi.org/10.1186/s12966-017-0561-4.

[26] Sullivan, A.N and Lachman, M.E 2016. Behavior Change with Fitness Technology in Sedentary Adults: A Review of the Evidence for Increasing Physical Activity. Frontiers in public health. 4, (2016), 289. DOI:https://doi.org/10.3389/fpubh.2016.00289.

[27] Sutton, S. 2008. How does the Health Action Process Approach (HAPA) bridge the intention-behavior gap? An examination of the model's causal structure. Applied Psychology. 57, 1 (2008), 66-74. DOI:https://doi.org/10.1111/j.1464-0597.2007.00326.x.

[28] Taylor, W.C., Paxton, R.J., Shegog, R., Coan, S.P., Dubin, A., Page, T.F. and Rempel, D.M. 2016. Impact of Booster Breaks and Computer Prompts on Physical Activity and Sedentary Behavior Among Desk-Based Workers: A Cluster-Randomized Controlled Trial. Preventing chronic disease. 13, (Nov. 2016), E155. DOI:https://doi.org/10.5888/pcd13.160231.

[29] Tong, X., Gromala, D., Bartram, L. and Carpendale, S. 2015. Evaluating the Effectiveness of Three Physical Activity Visualizations - How People Perform vs . Perceive. In Personal Visualization: Exploring Data in Everyday Life. 1, (2015).

[30] Wang, Y., Duan, L., Mueller, J., Butscher, S. and Reiterer, H. 2016 "Fingerprints": Detecting meaningful moments for mobile health intervention. Proceedings of the 18th International Conference on Human-Computer Interaction with Mobile Devices and Services Adjunct, MobileHCI 2016 (2016).

[31] Wang, Y., Fadhil, A., Lange, J.-P. and Reiterer, H. 2017. Towards a Holistic Approach to Designing Theory-based Mobile Health Interventions. arXiv.org. (2017). DOI: arXiv:1712.02548v1

[32] Wang, Y., Pfeil, U. and Reiterer, H. 2016. Supporting self-assembly: The IKEA effect on mobile health persuasive technology. MMHealth 2016 Proceedings of the 2016 ACM Workshop on Multimedia for Personal Health and Health Care, co-located with ACM Multimedia 2016 (2016). DOI: 10.1145/2985766.2985775.

[33] Wang, Y., Sommer, B., Schreiber, F. and Reiterer, H. 2018. Clustering with Temporal Constraints on Spatio-Temporal Data of Human Mobility. arXiv.org. (2018). DOI: arXiv:1807.00546v1.

[34] Wang, Y., Wu, L., Lange, J.-P., Fadhil, A. and Reiterer, H. 2017. Persuasive Technology in Reducing Prolonged Sedentary Behavior at Work: A
Systematic Review. Smart Health. (2018). DOI: https://doi.org/10.1016/j.smhl.2018.05.002. 